Research Paper

\title{
Submerged fermentation of Lactobacillus rhamnosus YS9 for $\gamma$-aminobutyric acid (GABA) production
}

\author{
Qian Lin \\ School of Life Science and Technology, Yulin Normal University, Yulin, Guangxi, P.R. China.
}

Submitted: July 4, 2010; Approved: July 2, 2012.

\begin{abstract}
$\gamma$-Aminobutyric acid (GABA) is a major inhibitory neurotransmitter in central nervous system, and its application in drugs and functional foods has attracted great attention. To enhance production of $\gamma$-aminobutyric acid, Lactobacillus rhamnosus YS9, a strain isolated from Chinese traditional fermented food pickled vegetable, was grown under submerged fermentation. Its cultivation conditions were investigated. When culture $\mathrm{pH}$ condition was adjusted to the optimal $\mathrm{pH}$ of glutamate decarboxylase activity, culture of Lb. rhamnosus YS9 in medium supplemented with $200 \mathrm{mM}$ of monosodium glutamate and $200 \mu \mathrm{M}$ of pyridoxal phosphate (PLP), produced $187 \mathrm{mM}$ of GABA.
\end{abstract}

Key words: Lactobacillus rhamnosus, $\gamma$-aminobutyric acid (GABA), glutamate decarboxylase (GAD).

\section{Introduction}

$\gamma$-aminobutyric acid (GABA), a ubiquitous non-protein amino acid, functions as a major depressive neurotransmitter in animals (Mody et al., 1994) and plays an important role in cardiovascular function (DeFeudis, 1983). Other physiological functions of GABA include induction of hypotensive, diuretic effects, tranquilizer effects and prevention of diabetic conditions (Jakobs et al., 1993; Wong et al., 2003). Dut to its multiple functions, GABA can be applied to production of many drugs and functional foods.

Currently, production of fermented food containing high level of GABA is actively pursued. Some microorganisms with high GABA-producing ability have been isolated, such as Monascus (Kono and Himeno, 2000; Su et al., 2003), Rhizopus (Aoki et al., 2003), Lactobacillus paracasei (Komatsuzaki et al., 2005), Lactobacillus brevis (Li et al., 2010; Park and Oh, 2007; Yokoyama et al., 2002), Lactococcus lactis (Nomura et al., 1998), Streptococcus thermophilus (Yang et al., 2008). Glutamate decarboxylase (GAD) is responsible for GABA synthesis in microorganisms. Using lactic acid bacteria (LAB) harboring GAD to produce GABA-enriched food has attracted great attention since they are safe and recognized as probiotics.

Lactobacillus rhamnosus is an important industrial LAB for its ability to produce high level of lactic acid. In addition, it is a promising probiotic bacterium because it has been reported to persist in the colon and modify the colonic environment with potential positive health effects (Goldin et al., 1992; Ling et al., 1994), and Lb. rhamnosus has been commercially used as starter of yoghourt production. However, there has been no investigation on fermentation of $L b$. rhamnosus for GABA production. In previous work, Lb. rhamnosus YS9 was isolated from Chinese traditional fermented food pickled vegetable, and it appeared to produce high yield of GABA. The culture conditions for this strain to produce high level of GABA were firstly investigated in this study.

\section{Materials and Methods}

\section{Strain, medium and culture conditions}

Lactobacillus rhamnosus YS9, a strain isolated from Chinese traditional fermented food pickled vegetable, was used throughout this study. The stock culture of $L b$. rhamnosus YS9 was reactivated by two or three successive 
transfers in $12 \%(\mathrm{w} / \mathrm{v})$ sterilized skim milk. For GABA production, the culture was subsequently inoculated into $1 \mathrm{~L}$ of flask containing $400 \mathrm{~mL}$ of nutrient medium, which consisted of following compositions (per liter): $20 \mathrm{~g}$ of glucose, $10 \mathrm{~g}$ of peptone, $10 \mathrm{~g}$ of beef extract, $5 \mathrm{~g}$ of yeast extract, $2 \mathrm{~g}$ of ammonium dibasic citrate, $2 \mathrm{~g}$ of sodium acetate, $2 \mathrm{~g}$ of $\mathrm{K}_{2} \mathrm{HPO}_{4}, 0.2 \mathrm{~g}$ of $\mathrm{MgSO}_{4} 7 \mathrm{H}_{2} \mathrm{O}, 0.05 \mathrm{~g}$ of $\mathrm{MnSO}_{4} \mathrm{H}_{2} \mathrm{O}$, $1 \mathrm{~mL}$ of tween $80,100 \mu \mathrm{M}$ of pyridoxal 5'-phosphate (PLP), pH6.5. Unless otherwise stated, the strain was grown at $37^{\circ} \mathrm{C}$. Monosodium glutamate (MSG) was added to broth at concentrations indicated in figure legends. In $\mathrm{pH}$-regulated cultivation, $\mathrm{pH}$ was adjusted every $12 \mathrm{~h}$ by adding $\mathrm{NaOH}$ or $\mathrm{HCl}$ during cultivation.

\section{Analysis of GABA and GAD activity assay}

Extracellular and intracellular GABA analysis and GAD activity assay were carried out as previously described (Yang et al., 2008). One unit of GAD activity was defined as the amount of enzyme that produced $1 \mu \mathrm{mol}$ of GABA in 1 min.

\section{Results and Discussion}

\section{Time course study of extracellular and intracellular GABA content}

A time course analysis of intracellular and extracellular GABA content in culture medium was conducted to assess the GABA-producing ability of L. rhamnosus YS9. Figure 1 shows the difference between extracellular and intracellular GABA content. Extracellular GABA was far higher than intracellular GABA, and the former peak appeared later than the latter. The reason for the phenomenon was that synthesis of GABA occurred intracellularly and GABA was secreted, accumulated extracellularly, which was consistent with the localization of GAD in cytoplasm of Lactobacillus (Higuchi et al., 1997). The peak of GABA production was much later than that of cell growth, which

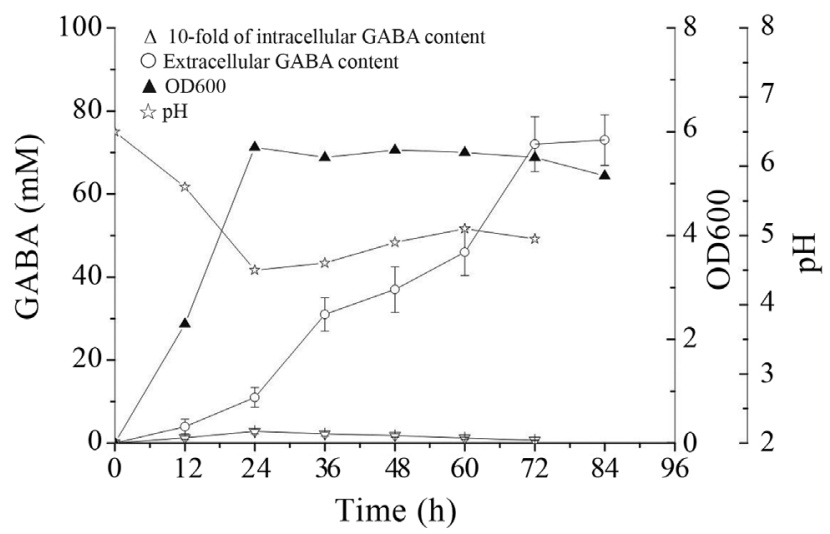

Figure 1 - Changes in extracellular and intracellular GABA content produced by Lb. rhamnosus YS9 grown in the medium containing $100 \mathrm{mM}$ of monosodium glutamate at $37^{\circ} \mathrm{C}$. The results were expressed as mean $\pm \mathrm{SD}$ from three independent experiments. might reflect that the enzyme responsible for GABA synthesis, glutamate decarboxylase (GAD), was induced by environmental stress. During growth, the decreased $\mathrm{pH}$ might be resulted from the strain's acid-producing ability which was well known as an important characteristic of LAB.

\section{Effect of MSG concentration and cultivation temperature on GABA production and cell growth}

For high level of GABA production by $L b$. rhamnosus YS9, optimal MSG concentration and cultivation termperature were determined by analysis of GABA content and cell optical density at various MSG concentrations and temperatures. Increasing concentration of MSG led to increasing GABA content (Figure 2) and the highest extracellular GABA yield was observed with $200 \mathrm{mM}$ of MSG, although cell growth rate seemed to be mildly inhibited (Figure 3). So it can be deduced that the GABA production may be improved with increased concentration of MSG added to the culture medium, and the MSG concentration above a certain level would inhibit cell growth. As for L. rhamnosus YS9, MSG concentration should be limited to $200 \mathrm{mM}$.

The relation of culture temperature and GABA production was examined (Figures 4 and 5). Within the range of test temperatures, cultivation at $43{ }^{\circ} \mathrm{C}$ led to the highest GABA yield and cell growth. So the optimal cultivation temperature of Lb. rhamnosus YS9 for GABA production was $43{ }^{\circ} \mathrm{C}$, which would drastically inhibit other LAB's growth rate, such as Lb. paracasei NFRI 7415 (Komatsuzaki et al., 2005). This might be due to different physiological and enzymatic characteristics between different GABA-producing LAB strains.

\section{Effect of PLP addition on extracellular GABA production}

GAD belonged to PLP-dependent decarboxylase, which would form Schiff's base with substrate, thus as a

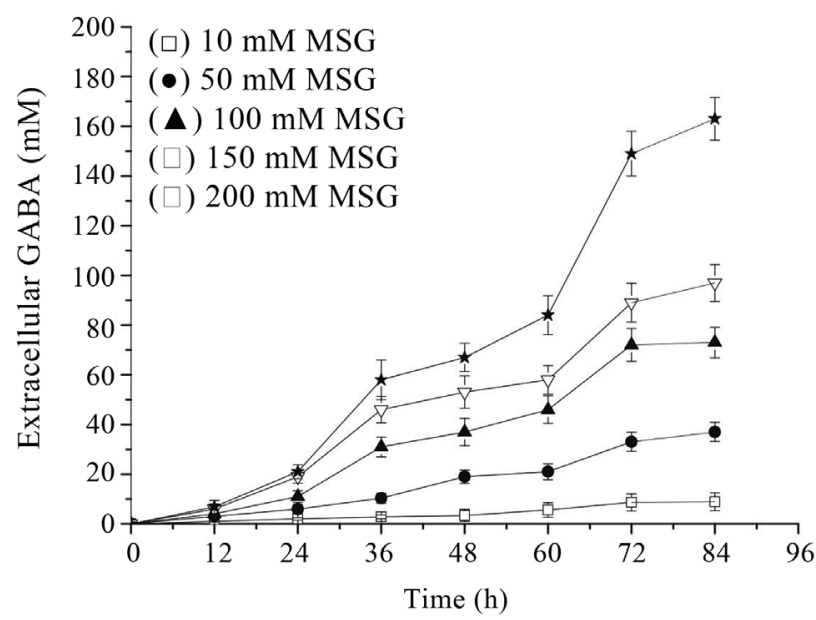

Figure 2 - Effect of monosodium glutamate concentration on extracellular GABA production. The results were expressed as mean \pm SD from three independent experiments. 


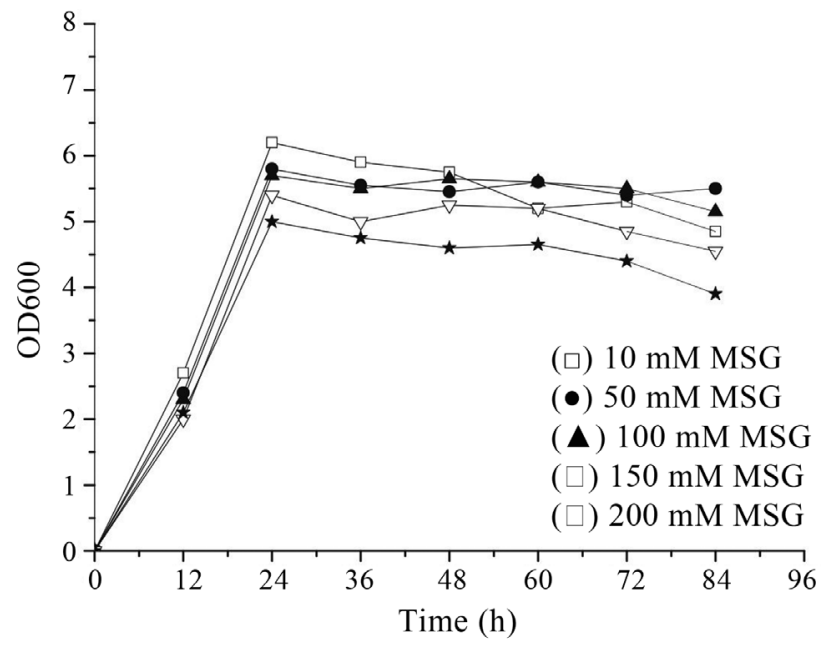

Figure 3 - Effect of monosodium glutamate concentration on cell growth of $L$ b. rhamnosus YS9.

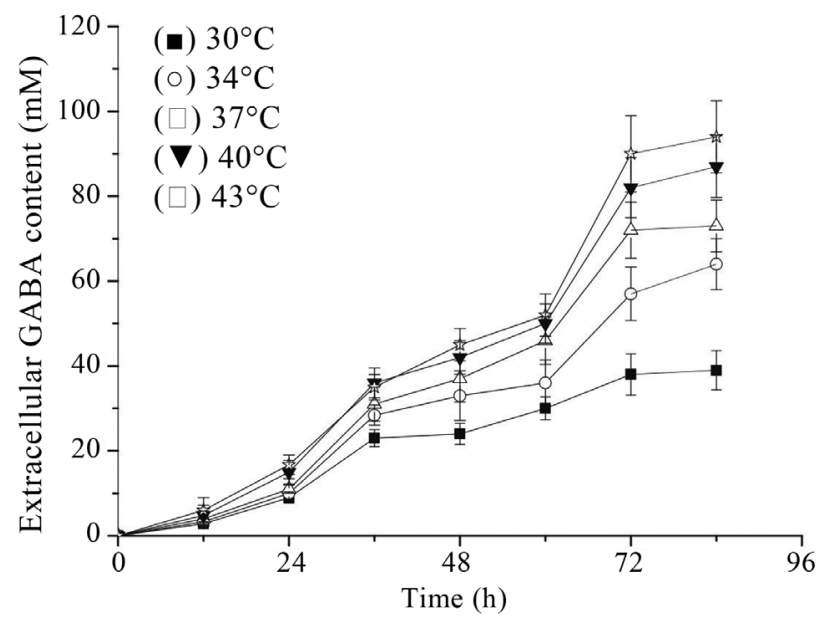

Figure 4 - Effect of culture temperature on extracellular GABA production. Lb. rhamnosus YS9 was grown in medium containing $100 \mathrm{mM}$ of MSG. The results were expressed as mean \pm SD from three independent experiments.

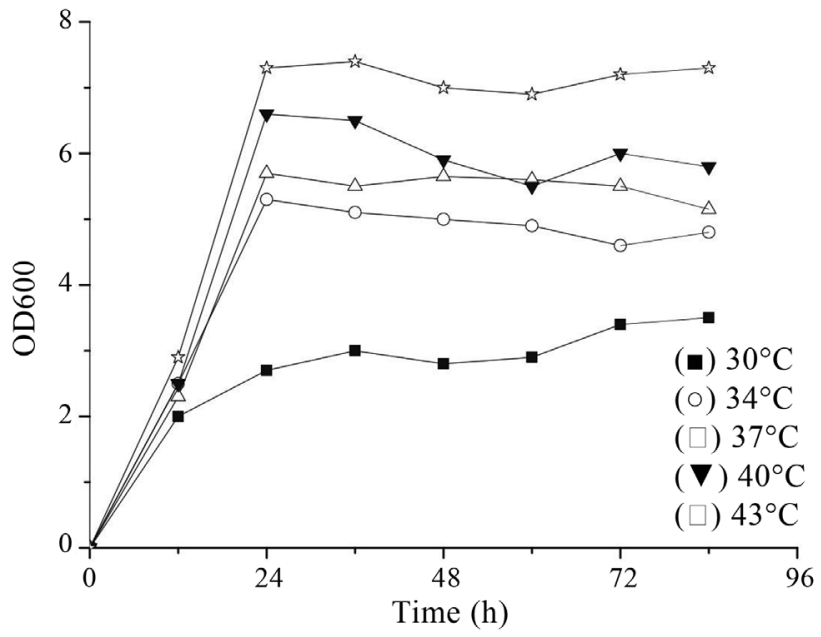

Figure 5 - Effect of culture temperature on cell growth. L. rhamnosus YS9 was grown in medium containing $100 \mathrm{mM}$ of MSG. coenzyme, PLP was essential to the enzyme activity. So it might be hypothesized that addition of PLP to the culture medium would improve GABA production. The effect of PLP addition was examined by analysis of extracellular GABA content in culture broth of L. rhamnosus YS9 with PLP at different concentrations. Figure 6 shows that the higher the concentration of PLP was, the higher level of extracellular GABA the strain would produce. In this study, PLP was added at initial cultivation, and the enhancement effect of GABA production was similar to that of $L$. paracasei NFRI 7415 (Komatsuzaki et al., 2005), but different from that of $S$. thermophilus Y2 (Yang et al., 2008). So the optimal concentration of PLP added to culture medium was $200 \mu \mathrm{M}$.

\section{Effect of $\mathrm{pH}$ regulation of culture medium on extracellular GABA production}

Many reports on bacterial GAD suggested that $\mathrm{pH}$ played an important role in the enzyme activity and GABA production. Since GABA synthesis was dependent on GAD, the highest yield of GABA might be obtained when the cultivation conditions were the same as that of enzyme catalysis. For the GAD of Lb. rhamnosus YS9, the optimal $\mathrm{pH}$ was determined by measurement of enzyme activity in cells under various $\mathrm{pH}$ conditions. GAD activity dramatically decreased at $\mathrm{pH} 4.0$ and $\mathrm{pH}>4.8$ (Figure 7). Lb. rhamnosus YS9 did not show high GAD activity at low $\mathrm{pH}$, although it was believed that bacterial GAD plays a major role in acid-resistance mechanism. As Figure 7 shows, the optimum $\mathrm{pH}$ for GAD activity was 4.4. Accordingly, GABA production was measured by cultivation of Lb. rhamnosus YS9 under $\mathrm{pH}-$ regulated conditions. Figure 8 shows that under $\mathrm{pH}$-regulated conditions and other optimized conditions $\left(43^{\circ} \mathrm{C}, 200 \mu \mathrm{M}\right.$ PLP, $200 \mathrm{mM}$ MSG), Lb. rhamnosus YS9 produced higher level of GABA,

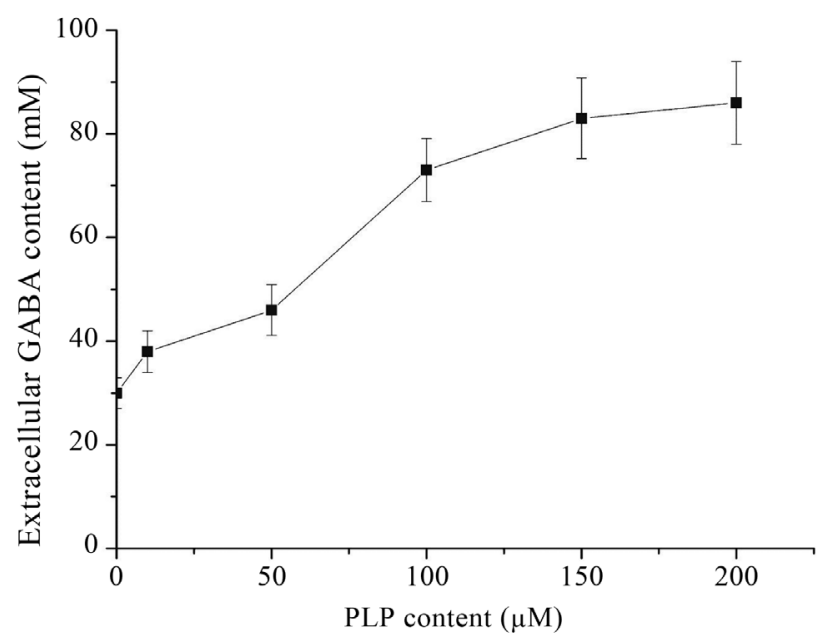

Figure 6 - Effect of PLP addition on extracellular GABA production. $L$. rhamnosus YS9 was grown at $37^{\circ} \mathrm{C}$ for $84 \mathrm{~h}$ in medium containing PLP at different concentrations and $100 \mathrm{mM}$ of MSG. The results were expressed as mean $\pm \mathrm{SD}$ from three independent experiments. 


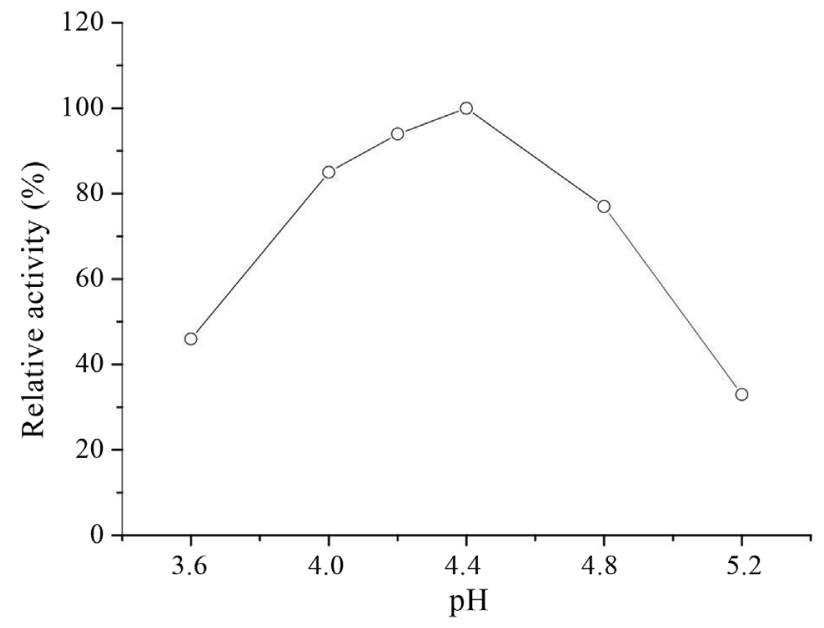

Figure 7 - Effect of $\mathrm{pH}$ on GAD activity in biotransformation of cells. GAD activity was measured at different $\mathrm{pH}$ and $43{ }^{\circ} \mathrm{C}$ for $1 \mathrm{~h}$. The data were expressed as mean from two independent experiments.

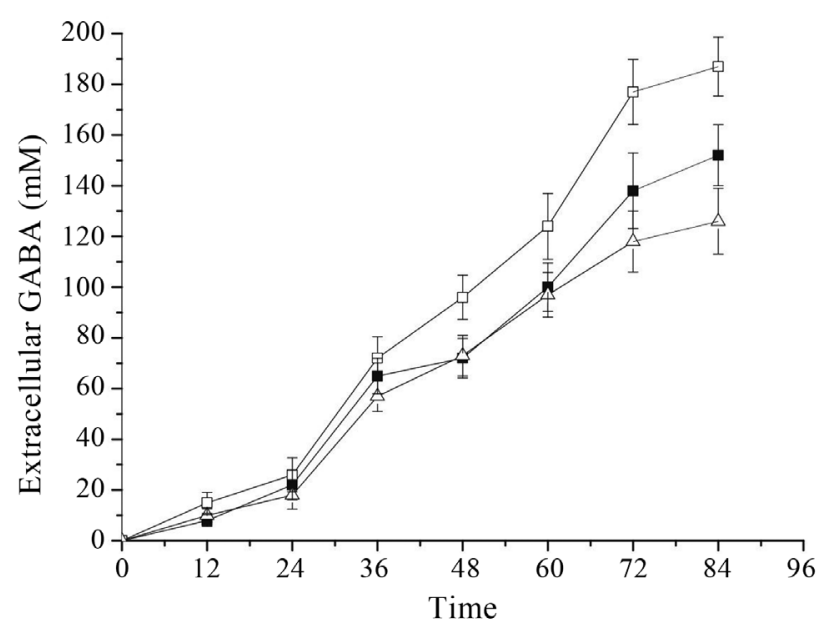

Figure 8 - Effect of $\mathrm{pH}$ regulation on extracellular GABA production. $L b$. rhamnosus YS9 was grown at $43{ }^{\circ} \mathrm{C}$ in medium containing $200 \mathrm{mM}$ of MSG and $200 \mu \mathrm{M}$ of PLP. The results were expressed as mean \pm SD from three independent experiments.

$187 \mathrm{mM}$, at $\mathrm{pH} 4.4$ than those at both $\mathrm{pH} 4$ and $\mathrm{pH} 4.8$, which might be due to the $\mathrm{pH}$ condition consistent with the optimal $\mathrm{pH}$ for GAD activity.

In summary, a strain of $L b$. rhamnosus was examined for its ability to produce GABA. Generally, bacterial glutamate decarboxylases are located in cytoplasm, whereas glutamate and GABA can be transported through membrane and exchanged, which results in the extracellular accumulation of GABA. So production of GABA can be achieved by cultivation of LAB under the GAD optimal reaction conditions. In this paper, by adjusting the culture conditions of $L b$. rhamnosus YS9 to achieve the GAD maximum activity, GABA production was enhanced dramatically. The yield of Lb. rhamnosus YS9, $187 \mathrm{mM}$, was at an intermediate level compared with those of $L b$. brevis IFO
12005, 10.18 mM (Yokoyama et al., 2002), Lb. brevis NCL912, $345.83 \mathrm{mM}$ (Li et al., 2010), Lb. paracasei NFRI 7415, $302 \mathrm{mM}$ (Komatsuzaki et al., 2005), and Streptococcus thermophilus Y2, $77 \mathrm{mM}$ (Yang et al., 2008). Though Lb. rhamnosus has been used widely for production of fermented foods, there is no investigation on its GABA-producing ability before. The strain's potential for functional food production was worthy of further exploration.

\section{Acknowledgments}

This work was supported by a grant from Research Foundation for Advanced Talents of Yulin Normal University (No. G2009042), Guangxi Natural Science Foundation (No. 2011GXNSFB018046), Guangxi Feature Specialty and Curriculum Integration Project (No. GXTSZY220, GXTSZY022).

\section{References}

Aoki H, Uda I, Tagami K, Furuya Y, Endo Y, Fujimoto K (2003) The production of a new tempeh-like fermented soybean containing a high level of $\gamma$-aminobutyric acid by anaerobic incubation with Rhizopus. Biosci. Biotechnol Biochem 67:1018-1023.

DeFeudis FV (1983) $\gamma$-Aminobutyric acid and cardiovascular function. Cell Mol Life Sci 39:845-849.

Goldin BR, Gorbach SL, Saxelin M, Barakat S, Gualtieri L, Salminen S (1992) Survival of Lactobacillus species (strain GG) in human gastrointestinal tract. Dig Dis Sci 37:121128.

Higuchi T, Hayashi H, Abe K (1997) Exchange of glutamate and $\gamma$-aminobutyrate in a Lactobacillus strain. J Bacteriol 179:3362-3364.

Jakobs C, Jaeken J, Gibson KM (1993) Inherited disorders of GABA metabolism. J Inher Metab Dis 16:704-715.

Komatsuzaki N, Shima J, Kawamoto S, Momose H, Kimura T (2005) Production of $\gamma$-aminobutyric acid (GABA) by Lactobacillus paracasei isolated from traditional fermented foods. Food Microbiol 22:497-504.

Kono I, Himeno, K. (2000). Changes in $\gamma$-aminobutyric acid content during beni-koji making. Biosci Biotechnol Biochem 64:617-619.

Li H, Qiu T, Cao Y, Yang J, Huang Z (2010) Medium optimization of gamma-aminobutyric acid by Lactobacillus brevis NCL912. Amino Acids 38:1439-1445.

Ling WH, Korpela R, Mykkanen H, Salminen S, Hanninen O (1994) Lactobacillus strain GG supplementation decreases colonic hydrolytic and reductive enzyme activities in healthy female adults. J Nutr 124:18-23.

Mody I, De Koninck Y, Otis TS, Soltesz I (1994) Bridging the cleft at GABA synapses in the brain. Trends Neurosci 17:517-525

Nomura M, Kimoto H, Someya Y, Furukawa S, Suzuki I (1998) Production of $\gamma$-aminobutyric acid by cheese starter during cheese ripening. J Dairy Sci 81:1486-1491.

Park KB, Oh SH (2007) Production of yogurt with enhanced levels of gamma-aminobutyric acid and valuable nutrients using lactic acid bacteria and germinated soybean extract. Bioresource Technol 98:1675-1679. 
Su YC, Wang JJ, Lin TT, Pan TM (2003) Production of the secondary metabolites $\gamma$-aminobutyric acid and monacolin K by Monascus. J Ind Microbiol Biotechnol 30:41-46.

Wong CG, Bottiglieri T, Snead OC (2003) GABA, $\gamma$-hydroxybutyric acid, and neurological disease. Ann Neurol 54:S3S12.

Yang SY, Lü FX, Lu ZX, Bie XM, Jiao Y, Sun LJ, Yu B (2008) Production of $\gamma$-aminobutyric acid by Streptococcus salivarius subsp. thermophilus Y2 under submerged fermentation. Amino Acids 34:473-478.

Yokoyama S, Hiramatsu J, Hayakawa K (2002) Production of $\gamma$-aminobutyric acid from alcohol distillery lees by Lactobacillus brevis IFO-12005. J Biosci Bioeng 93:9597.

All the content of the journal, except where otherwise noted, is licensed under a Creative Commons License CC BY-NC. 